Picture in Tropical Medicine and Health

\title{
Diphtheria-like Illness due to Corynebacterium Ulcerans infection
}

\author{
Nimfa Putong ${ }^{1}$, Gladys Agustin ${ }^{1}$, Monica Pasubillo ${ }^{1}$, Kei Miyagi ${ }^{2}$ \\ and Efren M. Dimaano ${ }^{1 *}$ \\ Received 30 August, 2010 Accepted 28 December, 2010 Published online 10 February, 2011
}

A 15 year-old Filipino male was admitted to San Lazaro Hospital, Manila, Philippines for the first time on last June 9, 2009 complaining of fever and difficulty breathing. His condition started 3 days prior to admission as high grade fever accompanied by productive cough, chills and sore throat with painful swallowing (oedynophagia). Self medication with paracetamol and phenylpropanolamine was done. A day later, the patient developed gradual swelling on both sides of the neck and difficulty breathing and hence sought treatment in our hospital. Pertinent physical examination findings include: BP 110/70mmHg, PR 94/min RR 28/min, presence of diffuse, tender swelling on both sides of the neck (bull? neck) and presence of a grayish-white membrane on the left tonsillar area extending to the uvula and edges of the soft palate. Past medical history revealed no immunization since birth other than BCG.

The patient was admitted as a case of diphtheria and treated with Penicillin G at 3 million IU every 4 hours IV push and Dexamethasone in the absence of Anti-Diphtheria Serum

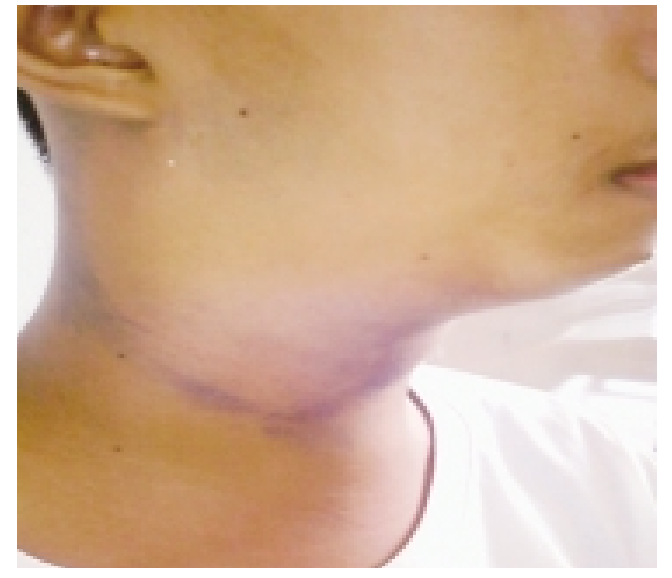

(no longer available in the market). Dexamethasone was given due to massive swelling of the tonsillar area causing severe dyspnea, $8 \mathrm{mg}$ initially then $4 \mathrm{mg}$ every 6 hours for 3 days. Throat swab and culture revealed Corynebacterium ulcerans. ECG revealed bradyarrythmia. Troponin test was positive and 2-D Echo with Doppler showed minimal posterior pericardial effusion; ejection fraction $26 \%$.

The patient was found to have nasal twang and gradual clearing of pseudomembrane with decreased size of bull neck, but later suffered severe chest pain and went into cardiac arrest. Despite resuscitative measures the patient expired on the 29th day after hospital admission.

Droplet transmission may be the cause of the infection because the patient had apparently not received any diphtheria vaccine since childhood. The cause of death is most probably due to acute fulminant myocarditis after diphtheria.

A total of 28 fatal cases of Diphtheria-like Illness due to Corynebacterium Ulcerans infection have been reported over the past 37years [1]

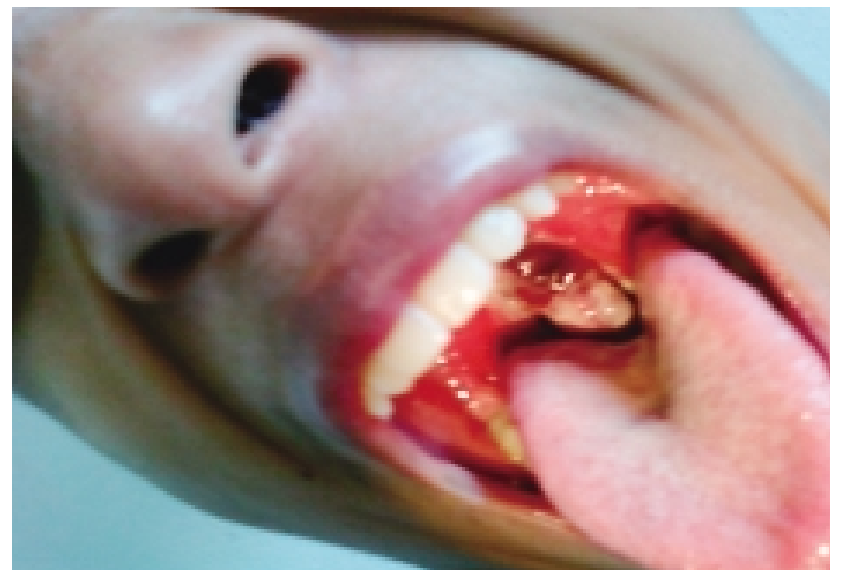

Figure. The bull neck appearance and pseudomembrane generally seen in diphtheria can also be manifested in Corynebacterium Ulcerans infection

\footnotetext{
San Lazaro Hospital, Department of Health, Manila, Philippines

Department of Clinical Medicine, Institute of Tropical Medicine, Nagasaki University, Japan

*Corresponding author

E-mail: dimaano.efren@yahoo.com
} 


\section{REFERENCES}

1 . Tiwari TS, Golaz A, Yu DT, Ehresmann KR, Jones TF, Hill HE, Cassiday PK, Pawloski LC, Moran JS, Popovic T, Wharton M. Investigations of 2 cases of diphtheria-like illness due to toxigenic Corynebacterium ulcerans Clin Infect Dis 2008:46:395-401 